\title{
On extensions of 1 chains
}

\author{
by \\ ERIK Dofs (Stockholm)
}

Introduction. Mohanty [2] introduced 1 chains and gave several fundamental theorems for 1 chains with positive integer elements. We now generalize the notion of 1 chain by extending the domain of definition:

Definitions. A sequence of integers $\left\{u_{i}\right\}$ in $K(\sqrt{-3})$, with at least three terms, in which any three consecutive terms satisfy the relation $u_{n-1} \cdot u_{n+1}=$ $u_{n}^{3}+1$, is called a 1 chain. A 1 chain that contains only rational integers is called a $1+$ chain if all elements are positive integers and a $1 \pm$ chain if it contains at least one negative integer.

In this paper we prove results for 1 chains, similar to Mohanty's [2] for $1+$ chains and some new ones concerning the least element(s) of a 1 chain.

We first solve the two open problems from Mohanty's paper [2]:

Is there any pair (of positive integers) $\{x, y\}$ such that $x \mid y^{2}-y+1$ and $y \mid x^{3}+1$ which cannot be obtained from $\{1,1\}$ by using the algorithm $x^{\prime}=\left(y^{2}-y+1\right) / x$ and $y^{\prime}=\left(x^{\prime 3}+1\right) / y$ (or this algorithm starting with $y$ instead)?

The answer is affirmative and the numerically smallest examples are $\{49,325\}$ and $\{49,362\}$, where each pair consists of consecutive terms in the $1+$ chain $\langle 325,49,362\rangle$ (where $\langle u, s, t\rangle$ stands for $\ldots, u, s, t, \ldots$ ). To see that these pairs cannot be obtained from $\{1,1\}$ it is sufficient to realize that the algorithm is reversible and generates a unique smallest pair independent of the order of execution. Other examples of $1+$ chains that contain such (smallest) pairs are $\langle 398,93,2021\rangle,\langle 962,147,3302\rangle$ and $\langle 2110,619,112406\rangle$.

The second open problem was:

Are there two (non-identical) $1+$ chains that have the same least element?

All $1+$ chains in which the least element is $\leq 78567$ are given in Table 1 and clearly there is just one instance: $\langle 3002,1209,588665\rangle$ and $\langle 13205,1209,133826\rangle$. 
Extension to rational integers. We now study $1 \pm$ chains and define the least element as the single element (except in a singular case) with the least absolute value. If we permit both $1+$ chains and $1 \pm$ chains in the second problem above, there are numerous examples of solutions including some of higher order:

$$
\begin{array}{ll}
s=-273:\langle-508,-273,40052\rangle,\langle-646,-273,31496\rangle, \\
\quad\langle-1054,-273,19304\rangle,\langle-2356,-273,8636\rangle ; \\
s=-9: \quad\langle-13,-9,56\rangle,\langle-28,-9,26\rangle,\langle-52,-9,14\rangle ; \\
s=-7: \quad\langle-9,-7,38\rangle,\langle-18,-7,19\rangle ; \\
s=31: \quad\langle-32,31,-931\rangle,\langle-56,31,-532\rangle,\langle-98,31,-304\rangle ; \\
s=65: \quad\langle 114,65,2409\rangle,\langle-66,65,-4161\rangle,\langle-146,65,-1881\rangle ; \\
s=1209:\langle 13205,1209,133826\rangle,\langle 3002,1209,588665\rangle, \\
& \langle-1210,1209,-1460473\rangle,\langle-4345,1209,-406714\rangle .
\end{array}
$$

In fact, for each $1+$ chain (or $1 \pm$ chain) $\langle u, s, t\rangle$ where $s>0$ is the least element, there is a $1 \pm$ chain $\left\langle-(s+1), s,-\left(s^{2}-s+1\right)\right\rangle$ with the same least element.

Mohanty studied the properties of $1+$ chains $\langle u, s, t\rangle$ in which $s \mid t+1$ or $s \mid t^{2}-t+1$ and proved that in these cases $u, s$ or $t$ is the least element. These results are valid also for $1 \pm$ chains with minor changes (proofs omitted) and the statement corresponding to (f) in [2] for $1 \pm$ chains is

(a) If $\langle u, s, t\rangle$ is a $1 \pm$ chain that does not have 0 as its least element and $s \mid t+1$, then $u, s$ or $t$ is the least element.

The excluded $1 \pm$ chains are singular, of the type $\left\langle-\left(M^{3}+1\right), M,-1\right.$, $0,-1$ for any $M \neq 0,-1$ as the next element is undefined. This singularity can be removed with a proper definition of $0 / 0$ but then the statement is not valid if $s=M$ in $\left\langle-\left(M^{3}+1\right), M,-1\right\rangle$.

Theorem 4 in [2] is valid for $1 \pm$ chains if $s \neq 0$ :

(b) If $\langle u, s, t\rangle$ is a $1 \pm$ chain, then $s \neq 0$ is the least element if and only if $|s|<|t|<s^{2}$.

To make Theorem 6 in [2] valid for $1 \pm$ chains, we have to make a minor exception:

(c) If $s \neq 0$ and $s \mid t^{2}-t+1$ in $\langle u, s, t\rangle$, then $u, s$ or $t$ is the least element except if $\langle u, s, t\rangle=\left\langle-t^{5}+3 t^{4}-6 t^{3}+7 t^{2}-6 t+3,-\left(t^{2}-t+1\right), t\right\rangle$ and $t<0$.

All $1+$ chains and $1 \pm$ chains that satisfy both $u \mid s^{2}-s+1$ and $s \mid u^{2}-u+1$ for some consecutive elements $u, s$ were given by Mills [1]: the $1+$ chain $\langle 1,1\rangle$ and two infinite sets of $1 \pm$ chains with $\langle u, s\rangle=\left\langle\alpha_{n}, \alpha_{n+1}\right\rangle$ satisfying $\alpha_{n+1}=$ $5 \alpha_{n}-\alpha_{n-1}+1, \alpha_{0}=\alpha_{1}=-1,\left\langle\beta_{n}, \beta_{n+1}\right\rangle$ where $\beta_{n+1}=-3 \beta_{n}-\beta_{n-1}+1$ 
and $\beta_{0}=1, \beta_{1}=-1 . \alpha_{n}$ and $\beta_{n}$ are the least elements in their respective $1 \pm$ chains except if 0 is the least element or $\langle u, s\rangle=\left\langle\alpha_{2}, \alpha_{3}\right\rangle=\langle-3,-13\rangle$, in accordance with statement (c).

1 chains with common elements. In this section we consider only 1 chains with rational integers and show some interesting connections between such 1 chains that have common elements; first the simple but fundamental

TheOREM A. If $\left\langle u_{1}, s\right\rangle$ and $\left\langle u_{2}, s\right\rangle$ are two 1 chains, $f$ a factor of $u_{1} u_{2}$ that satisfies $f^{3}+1 \equiv 0(\bmod s)$ and $\left(u_{1} u_{2} / f\right) \mid s^{3}+1$, then there is a third 1 chain $\langle u, s\rangle$ with $u=u_{1} u_{2} / f$.

Pr o o f. By assumption we have $u=\left(u_{1} u_{2} / f\right) \mid s^{3}+1$. Further, $u_{1}^{3} \equiv u_{2}^{3} \equiv$ $f^{3} \equiv-1(\bmod s)$, leading to $\left(u_{1}^{3} u_{2}^{3} / f^{3}\right) \equiv-1(\bmod s)$ or $\left(u_{1} u_{2} / f\right)^{3}+1 \equiv 0$ $(\bmod s)$.

Re m ark. $f=-1$ always satisfies the first divisibility condition and the second is satisfied if $u_{1} \mid s+1$ and $u_{2} \mid s^{2}-s+1$ or if $\left(u_{1}, u_{2}\right)=1$.

ExAmples. $\langle 26,-9\rangle,\langle 14,-9\rangle$ and $f=-1,2,-4,-7, \ldots$ gives the $1 \pm$ chains $\langle u,-9\rangle$ with $u=-364,182,-91,-52$, etc. The $1 \pm$ chain $\langle-7,19\rangle$ combined with itself $\left(u_{1}=u_{2}=-7, s=19, f=-1\right)$ generates $\langle-49,19\rangle$.

Corollary A. If $\langle u, s\rangle$ and $\left\langle u_{1}, s\right\rangle\left(u_{1} \neq 0\right)$ are 1 chains and $u_{1} \mid u$ then $\left\langle-u / u_{1}, s\right\rangle$ is a 1 chain.

Pr o of. The 1 chains $\langle u, s\rangle$ and $\left\langle\left(s^{3}+1\right) / u, s\right\rangle$ are identical though reversed. Apply Theorem A (with $f=-1)$ to $\left\langle\left(s^{3}+1\right) / u, s\right\rangle$ and $\left\langle u_{1}, s\right\rangle$ where $u_{1} \mid u$. Clearly $\left(\left(s^{3}+1\right) / u\right) \cdot u_{1}=\left(s^{3}+1\right) /\left(u / u_{1}\right) \mid s^{3}+1$ and thus $\left\langle\left(s^{3}+1\right) /\left(-u / u_{1}\right), s\right\rangle$ exists and is the (reversed) 1 chain $\left\langle-u / u_{1}, s\right\rangle$.

Theorem B. If $\langle u, s\rangle$ is a $1+$ chain in which $s$ is the least element and $s \mid u^{2}-u+1$, then $u$ is the least element in another $1+$ chain $\langle u, s(u+1)\rangle$.

Proof. By applying Theorem A (with $u$ as the common element and $f=$ $-1)$ to $\langle u,-(u+1)\rangle$ and $\langle u, s\rangle$ given that $s \mid u^{2}-u+1$, we see that $\langle u, s(u+1)\rangle$ is indeed another $1+$ chain and the element preceding $u$ is $\left(u^{2}-u+1\right) / s$. It remains to show that $u$ is the least element. Clearly $s(u+1)>u$. We know from [2] that $u=s$ if and only if $u=s=1$ in which case the theorem is true. If $s<u$ we immediately have $s \leq u-1<u-1+(1 / u)$, leading to $\left(u^{2}-u+1\right) / s>u$.

Corollary B. By Theorem 3(i) in [2] we see that Theorem B also can be used to generate the $1+$ chain $\langle t, s(t+1)\rangle$ with $t=\left(s^{3}+1\right) / u$, in which $t$ is the least element. 
EXAMPLES. $\langle 1,1\rangle$ generates the (same) $1+$ chain $\langle 2,1\rangle$, but the last choice of elements gives a new $1+$ chain $\langle 3,2\rangle$. $\langle 325,49,362\rangle$ generates the $1+$ chains $\langle 15974,325,2149\rangle$ and $\langle 17787,362,2667\rangle$.

Remark. Theorem B is also valid for $1 \pm$ chains, except for singular ones, but the proof requires more details.

A theorem that could simplify a search for additional solutions to Mohanty's second problem is

Theorem C. Let $\left\langle u_{1}, s\right\rangle$ be a $1+$ chain and $s$ its least element and assume there exists a $1 \pm$ chain $\left\langle u_{2}, s\right\rangle$, where $u_{2}<0$ and $\left|u_{2}\right|<s^{2} / u_{1}$. If also $u_{1} u_{2} \mid s^{3}+1$, then the $1+$ chain $\left\langle-u_{1} u_{2}, s\right\rangle$ exists and $s$ is also the least element of this $1+$ chain.

Proof. The existence of the $1+$ chain $\left\langle-u_{1} u_{2}, s\right\rangle$ follows directly from Theorem A. We have $s<-u_{1} u_{2}$ as $s<u_{1}$ and $u_{2}<0$. Further (by assumption), $-u_{1} u_{2}<s^{2}$, and consequently $s$ is the least element by Theorem 4 of $[2]$.

Remark. Also a combination of the $1+$ chain $\left\langle u_{1}, s\right\rangle$ with $0<u_{1}<s$ and the $1 \pm$ chain $\left\langle u_{2}, s\right\rangle$ where $u_{2}<0$ and $s$ the least element gives a $1+$ chain $\left\langle-u_{1} u_{2}, s\right\rangle$ with the same least element provided that $-u_{1} u_{2}<s^{2}$ and $u_{1} u_{2} \mid s^{3}+1$.

Parametric $1 \pm$ chains. In $[2$, Th. 2], all $1+$ chains and $1 \pm$ chains $\langle u, s\rangle$ were given in which $u \mid s+1$ for some consecutive elements: $\langle 1,1\rangle$, $\langle 2,3\rangle,\langle 5,9\rangle$, and the parametric ones: $\langle M,-(M+1)\rangle,\left\langle-M^{2}, M^{3}-1\right\rangle$ with $M \neq 0, \pm 1$, and $\langle-1, M\rangle$ (see (a) above).

It is more difficult to find parametric $1 \pm$ chains in which some consecutive elements satisfy $u \mid s^{2}-s+1$, apart from $\langle-1, M\rangle,\langle M,-(M+1)\rangle$ and 1 chains derived from them by means of Theorem 5 of [2] or its corollary, extended to $1 \pm$ chains. The smallest examples (with smallest degrees of polynomials) that we have found are: $\langle u, s\rangle=\left\langle-\left(M^{5}+2 M^{4}+5 M^{3}+\right.\right.$ $\left.\left.5 M^{2}+4 M+1\right),-\left(M^{4}+M^{3}+3 M^{2}+M+1\right)\right\rangle$ and $\left\langle M^{5}+4 M^{4}+9 M^{3}+\right.$ $\left.11 M^{2}+8 M+3,-\left(M^{4}+3 M^{3}+5 M^{2}+3 M+1\right)\right\rangle$, in which $s$ is the least element except if $s=-1$.

It is quite difficult to find parametric $1 \pm$ chains in the frequent case where $u \nmid s+1$ and $u \nmid s^{2}-s+1$ for all consecutive elements; however, one example is sufficient to prove

TheOREM D. There exist an infinite number of $1 \pm$ chains that neither satisfy $u \mid s+1$ nor $u \mid s^{2}-s+1$ for any consecutive elements.

Proof. For any $M(\neq 0, \pm 1)$, all consecutive elements in the $1 \pm$ chain $\left\langle-\left(M^{23}+4 M^{20}+10 M^{17}+16 M^{14}+19 M^{11}+17 M^{8}+10 M^{5}+4 M^{2}\right),-\left(M^{18}+\right.\right.$ 
$\left.\left.3 M^{15}+6 M^{12}+7 M^{9}+6 M^{6}+4 M^{3}+1\right)\right\rangle$ satisfy $u \nmid s+1$ and $u \nmid s^{2}-s$ +1 .

Extension to integers in $K(\sqrt{-3})$. If we permit the elements of a 1 chain to be integers in $K(\sqrt{-3})$, results similar to those in [2] and in this paper for $1 \pm$ chains can be obtained. This is due to the field $K(\sqrt{-3})$ being Euclidean and to the arithmetic structure of the 1 chain algorithm. However, most proofs require considerably more details than the corresponding theorems for 1 chains containing only real elements. Even the generalization of a fairly obvious statement like $(\mathrm{d})$ in [2] needs a proof:

TheOrem E. If $\left\langle u_{i}, u_{i+1}\right\rangle$ is a non-singular 1 chain and $\left|u_{i}\right|<\left|u_{i+1}\right|$, then $\left|u_{i+1}\right|<\left|u_{i+2}\right|$.

Proof.

$$
\begin{aligned}
\left|u_{i+2}\right| & =\left|\left(u_{i+1}^{3}+1\right) / u_{i}\right| \geq\left|u_{i+1}^{3} / u_{i}\right|-1 /\left|u_{i}\right| \\
& \geq\left|u_{i+1}^{2}\right|\left|u_{i+1} / u_{i}\right|-1>\left|u_{i+1}\right|^{2}-1>\left|u_{i+1}\right|
\end{aligned}
$$

as $\left|u_{i+1}\right|>\left|u_{i}\right| \geq 1$ implies $\left|u_{i+1}\right| \geq \sqrt{3}$.

In order to shorten several of the proofs below we state the simple:

LEMMA. If $\langle u, s\rangle$ is a 1 chain, then also $\langle u \mu, s \tau\rangle$ is a 1 chain if $\mu, \tau=1, \sigma$ or $\widehat{\sigma}$, where $\sigma^{3}=1$ and $\sigma \neq 1$ (we call such 1 chains associated).

The definition of least element, given above for $1 \pm$ chains, is easily extended to 1 chains with elements in $K(\sqrt{-3})$, but there are infinitely many 1 chains that have two least elements (see Theorem F).

Theorem 4 of [2] and (b) above, used in proofs concerning the least element, are not valid for all 1 chains with elements in $K(\sqrt{-3})$ :

THEOREM F. There are infinitely many 1 chains $\langle u, s, t\rangle$ that satisfy $|u|=|s|$ and $|t|=\left|\left(s^{3}+1\right) / s\right|>|s|^{2}$. In these 1 chains $s$ and $u$ are the least elements but $|t|<|s|^{2}$ is not satisfied, in contrast to (b).

Proof. If $s=a+(1-a) \sigma=\widehat{u}(a$ is a real integer $)$, then $|t|^{2}=|s|^{4}$ +3 .

Problem. In addition to the 1 chain given in Theorem $\mathrm{F},\langle-1,-1\rangle$ and associated 1 chains, is there any other 1 chain that satisfies $|u|=|s|$ ?

Remark. The problem is of some significance as it is possible to show that if $s$ is the least element in $\langle u, s, t\rangle$ and $|t| \geq|s|^{2}$ then $|u|=|s|$, except if $\langle u, s, t\rangle=\langle 2,1,1\rangle$ or an associated 1 chain.

Theorem B above is not generally true for 1 chains with elements in $K(\sqrt{-3})$ : 
EXAmples. Assume that $s$ is the least element in $\langle u, s\rangle$ and $s \mid u^{2}-u+1$. If $\langle u, s\rangle=\langle 2,1-\sigma\rangle,\langle 2,1-\widehat{\sigma}\rangle$ or if $s=-(u+\sigma)$ and $|u+\sigma| \leq|u|$ but $|u|>|u+\widehat{\sigma}|$, which is true for all integers of the form $u=1+r e^{i \theta}$ where $|\theta|<\pi / 6$, then $\left|\left(u^{2}-u+1\right) / s\right|<|u|$, which implies that $u$ is not the least element of $\langle s(u+1), u\rangle$.

The theorem corresponding to (f) in [2] and (a) above, can be stated similarly to (a):

TheOREM G. If $\langle u, s, t\rangle$ is a non-singular 1 chain, $u, s$ and $t$ are integers in $K(\sqrt{-3})$ and $s \mid u+1$, then also $s \mid t+1$ and $u, s$ or $t$ is the least element.

Proof. The first part is easy. To prove the second part put $u+1=\alpha s(\alpha$ an integer in $K(\sqrt{-3})$ ). If $N(\alpha) \geq 3$ we get $|s|=|u+1| /|\alpha| \leq(|u|+1) / \sqrt{3}$, implying $|s|<|u|$ unless $|u|=1$. However, $u \neq-1,-\sigma,-\widehat{\sigma}$ as the 1 chain is non-singular. $u=1$ implies $|\alpha|=2$, which gives the 1 chain $\langle 1,1\rangle$ (or associated ones) in which $u$ and $s$ are the least element. $u=\sigma, \widehat{\sigma}$ gives the contradiction $|\alpha|=1$. Define $\beta$ by $t+1=\beta$ s. If we also assume $N(\beta) \geq 3$ we get $|s|<|t|$ and $s$ is the least element, unless $t=1$ (or $u=1$ ). The remaining case $N(\alpha)=1$ (or $N(\beta)=1$ ) implies that $\alpha$ is a unit in $K(\sqrt{-3})$. Then $t=\left( \pm(u+1)^{3}+1\right) / u$ and either $u \mid 2$ or $t=-\left(u^{2}+3 u+3\right)$. If $u \mid 2$, a finite number of cases occur and $u$ or $s$ is the least element. The second case gives $\langle u, s, t\rangle=\left\langle u,-(u+1),-\left(u^{2}+3 u+3\right)\right\rangle$ (or an associated 1 chain), in which $u$ or $s$ is the least element. $\left|u^{2}+3 u+3\right|>|u+1|$ is false for some integer $u$-values (e.g. $u=\sigma-1$ ) but in these cases the 1 chain is singular.

Remark. The theorem is also true if $s \mid u+\sigma$ but in this case $s \mid t+\widehat{\sigma}$.

A generalization of Theorem 4 of [2] which takes Theorem $\mathrm{F}$ above into account is:

TheOREM H. If $\langle u, s, t\rangle$ is a non-singular 1 chain, $u, s, t$ are integers in $K(\sqrt{-3})$ and $s$ is a unique least element, i.e. $|u|>|s|$ and $|t|>|s|>1$, then $|u|<|s|^{2}$ and $|t|<|s|^{2}$. On the other hand, if $1<|s|<|u|<|s|^{2}$, then $|t| \geq|s|$, i.e. $s$ is a least (but not necessarily unique) element.

Proof. As $N(u)=|u|^{2}$ etc. we have to prove $N(u)<N\left(s^{2}\right)=N^{2}(s)$ in the first case. We know that $N(t)=N\left(\left(s^{3}+1\right) / u\right)=N\left(s^{3}+1\right) / N(u) \geq$ $N(s)+1$ as $|t|>|s|$. This gives $N(u) \leq N\left(s^{3}+1\right) /(N(s)+1)$. The triangle inequality implies $\left|s^{3}+1\right| \leq\left|s^{3}\right|+1$, i.e. $N\left(s^{3}+1\right) \leq N\left(s^{3}\right)+2 \sqrt{N\left(s^{3}\right)}+1$, leading to

$$
N(u) \leq \frac{N\left(s^{3}\right)+2 \sqrt{N\left(s^{3}\right)}+1}{N(s)+1}=\frac{N^{3}(s)+2 \sqrt{N^{3}(s)}+1}{N(s)+1}<N^{2}(s)
$$

for all integers $s$ with $N(s)>4$. No exceptions occur if $N(s)=3,4$. 
To prove the second part of the theorem: $|u|<|s|^{2}$ implies that

$$
N(t)=\frac{N\left(s^{3}+1\right)}{N(u)} \geq \frac{N\left(s^{3}+1\right)}{N^{2}(s)-1} \geq \frac{\left(\sqrt{N^{3}(s)}-1\right)^{2}}{N^{2}(s)-1} .
$$

Now

$$
N(t)-N(s) \geq \frac{-(2 N(s)+\sqrt{N(s)}+1)}{(N(s)+1)(\sqrt{N(s)}+1)}>-1
$$

if $N(s)>1$. Thus $N(t) \geq N(s)$ as the norms are (rational) integers.

We are now in a position to prove the theorem corresponding to Theorem 6 in [2] and (c) above:

THEOREM I. If $\langle u, s, t\rangle$ is a non-singular 1 chain and $s \mid u^{2}-u+1$, then $u, s$ or $t$ is the least element except if $s=-\left(u^{2}-u+1\right)$ or an associated integer and $|u+1|<|u|$.

Proof. We can assume $|s|>1$ as $s=-1,-\sigma,-\widehat{\sigma}$ would make the 1 chain singular and if $s=1, \sigma, \widehat{\sigma}$ the theorem is true. Put $u^{2}-u+1=\alpha s$ and assume first that $|u|^{2}<|s|$. Then

$$
|\alpha|<\left|\frac{u^{2}-u+1}{u^{2}}\right| \leq 1+\frac{1}{|u|}+\frac{1}{|u|^{2}}<\sqrt{3}
$$

if $|u| \geq \sqrt{7}$, i.e. $|\alpha|=1$. In the same way $|\alpha|=1, \sqrt{3}$ if $|u|=\sqrt{3}, 2$. Of these $\alpha$-values, $\alpha=-1,-\sigma,-\widehat{\sigma}$ give the exceptions in the theorem, while the case $|u|=1$ gives no exception.

Next, the case $|u|^{2}=|s|$ is impossible (unless $|s|=|u|=1$ ): If $r$ is the element preceding $u$ in $\langle u, s, t\rangle$ we have

$$
|u|-\frac{1}{|u|^{2}} \leq|r|=\left|\frac{u^{3}+1}{u^{2}}\right| \leq|u|+\frac{1}{|u|^{2}}
$$

and this gives

$$
(\sqrt{N(u)}-1 / N(u))^{2} \leq N(r) \leq(\sqrt{N(u)}-1 / N(u))^{2}
$$

or

$$
-\frac{2}{\sqrt{N(u)}}+\frac{1}{N^{2}(u)} \leq N(r)-N(u) \leq \frac{2}{\sqrt{N(u)}}+\frac{1}{N^{2}(u)} .
$$

$N(u)=3,4$ give no 1 chains while $N(r)=N(u)$ if $N(u) \geq 7$. If $|u|^{2}=|s|$ and $|r|=|u|$ then $|r s|=\left|u^{3}+1\right|=\left|u^{3}\right|$ but there is no integer $u$ in $K(\sqrt{-3})$ that satisfies this.

Finally, we have the case $|u|^{2}>|s|$. Now if $1<|u|<|s|, u$ is the least element by Theorem H. If $1<|u|=|s|$ then $u$ (and $s$ ) are the least element as 


$$
|t|=\left|\frac{s^{3}+1}{u}\right|=\left|\frac{s^{3}+1}{s}\right| \geq|s|^{2}-\frac{1}{|s|}>|s|,
$$

since $|s| \geq \sqrt{3}$. The same reasoning can be carried out for $t$ (instead of $u$ ) as $s \mid t^{2}-t+1$ according to the (easily) generalized Theorem 3 of [2].

The only remaining case is $|s|<|u|$ and $|s|<|t|$ but then obviously $s$ is the least element.

Finally, we prove the generalization of Theorem 2 of [2]:

Theorem J. All solutions of

$$
\alpha^{3}+\beta+1=\alpha \beta \Gamma,
$$

where $\alpha, \beta, \Gamma$ integers in $K(\sqrt{-3})$, are the following:

I. the (rational integer) solutions: $(1,1),(1,2),(2,1),(2,3),(2,9)$, $(3,2),(3,14),(5,9),(5,14)$;

II. the parametric solutions: $(\alpha,-1),(-1, \beta),(\alpha,-(\alpha+1))$, $\left(\alpha,-\left(\alpha^{2}-\alpha+1\right)\right),\left(\alpha,-\left(\alpha^{3}+1\right)\right),\left(-r^{2}, r^{3}-1\right)$ where $r$ is an integer in $K(\sqrt{-3})$;

III. $(1-2 \sigma,-2 \sigma),(1-2 \sigma,-9 \sigma),(-1+3 \sigma, 4-2 \sigma),(-1+3 \sigma,-9 \widehat{\sigma})$, $(-1+\sigma, 2),(-1+\sigma, 2+3 \sigma),(1+2 \sigma,-1-3 \sigma),(1+2 \sigma, 2)$;

IV. $(\alpha \sigma, \beta),(\alpha \widehat{\sigma}, \beta)$ and $(\widehat{\alpha}, \widehat{\beta})$ where $(\alpha, \beta)$ is any of the above solutions.

Proof. As in [2], put $r=(\beta+1) / \alpha, s=\left(\alpha^{3}+1\right) / \beta, t=r s-\alpha^{2}(=\Gamma)$, assuming $\alpha, \beta \neq 0$. Then $\alpha^{2}-\alpha r t+(r+t)=0$ and the discriminant

$$
(r t)^{2}-4(r+t)=u^{2}
$$

for some $u$, where $r, t, u$ are integers in $K(\sqrt{-3})$.

We note that if $(r, t)$ is a solution of $(2)$ corresponding to $(\alpha, \beta)$, then also $(r \sigma, t \sigma),(r \widehat{\sigma}, t \widehat{\sigma})$ and $(t, r)$ are solutions. The first two cases correspond to the solutions $(\alpha \widehat{\sigma}, \beta)$ and $(\alpha \sigma, \beta)$ while the third one corresponds to $\left(\alpha,\left(\alpha^{3}+1\right) / \beta\right)$. For sufficiently large (absolute) values of $r$ and $t$ the difference between $(r t)^{2}$ and adjacent squares becomes bigger than $4|r+t|$ and then $r+t$ must equal 0 . This gives $\alpha=-r^{2}$ and the solution $\left(-r^{2}, r^{3}-1\right)$. To determine the other solutions we assume $r+t \neq 0$ and get

$$
\begin{aligned}
2|r||t|-1 & \leq 2|r t|-1 \leq|r t+u||r t-u| \leq\left|r^{2} t^{2}-u^{2}\right| \\
& =4|r+t| \leq 4(|r|+|t|)
\end{aligned}
$$

or

$$
|r| \leq(4|t|+1) /(2|t|-4) \quad \text { if }|t|>2 .
$$

If also $|r|>2$ and $|t|=\sqrt{7}, 3,2 \sqrt{3}, \sqrt{13}, 4$ or $\sqrt{19}$ then it is sufficient to try all $r$-values with $|r| \leq \sqrt{79}, \sqrt{42}, 5, \sqrt{21}, 4, \sqrt{13}$ respectively, due to the symmetry of (2). No solutions occur in these cases and it remains to consider the cases $|r|=0,1, \sqrt{3}, 2$ (or $|t|$ assuming these values). 
$|r|=0$ leads to $t=-\alpha^{2}$ and $(\alpha, \beta)=(\alpha,-1)$ while $|t|=0$ gives the solution $\left(\alpha,-\left(\alpha^{3}+1\right)\right)$.

$|r|=1$ has two distinct subcases: $r=-1$ (or $-\sigma,-\widehat{\sigma}$ ) connected with $(\alpha, \beta)=(-1,0)$ and $(\alpha,-(\alpha+1))$, and $r=1$ (or $\sigma, \widehat{\sigma})$ which gives the solutions $(\alpha, \beta)=(1-2 \sigma,-2 \sigma),(2,1)$ and $(3,2) \cdot t=-1$ gives $(\alpha, \beta)=$ $(-1, \beta)$ and $\left(\alpha,-\left(\alpha^{2}-\alpha+1\right)\right)$ while $t=1$ gives $(1-2 \sigma,-9 \sigma),(2,9)$ and $(3,14)$.

$|r|=\sqrt{3}$ gives the remaining (III) solutions, while $|r|=2$ gives the remaining real integer solutions (I).

Acknowledgements. The author is greatly indebted to Hans Riesel for his continuous advice. He also verified or simplified some proofs and put extensive calculations on 1 chains at my disposal.

Table 1. $1+$ chains $\langle s, t\rangle ; s$ (least element) $<78568$

\begin{tabular}{|c|c|c|c|c|c|c|c|}
\hline$s$ & $t$ & $s$ & $t$ & $s$ & $t$ & $s$ & $t$ \\
\hline 1 & 1 & 2110 & 7189 & 8554 & 103211 & 39153 & 2431274 \\
\hline 2 & 3 & 2219 & 18702 & 8606 & 33069 & 39219 & 960890 \\
\hline 5 & 9 & 2345 & 11889 & 8909 & 110565 & 39302 & 3097413 \\
\hline 14 & 45 & 2394 & 37841 & 8918 & 179371 & 39654 & 7049315 \\
\hline 35 & 54 & 2449 & 52850 & 9083 & 566954 & 40369 & 3201341 \\
\hline 49 & 325 & 2763 & 44498 & 9425 & 184149 & 43225 & 1577749 \\
\hline 65 & 114 & 2881 & 25498 & 9603 & 13034 & 46298 & 760609 \\
\hline 93 & 398 & 2989 & 25070 & 9774 & 23579 & 46534 & 1974355 \\
\hline 99 & 626 & 3302 & 74149 & 10202 & 880859 & 47538 & 638009 \\
\hline 117 & 413 & 3365 & 138894 & 14315 & 24594 & 47619 & 4441766 \\
\hline 147 & 962 & 3377 & 83522 & 14363 & 56126 & 48650 & 327249 \\
\hline 185 & 434 & 3722 & 63729 & 14921 & 50661 & 50195 & 347634 \\
\hline 234 & 329 & 3794 & 74283 & 14949 & 646766 & 50861 & 94977 \\
\hline 299 & 3150 & 4074 & 71585 & 15419 & 1740918 & 52281 & 3476753 \\
\hline 325 & 2149 & 4257 & 158234 & 16213 & 989054 & 55734 & 213995 \\
\hline 362 & 2667 & 4406 & 15707 & 16254 & 330965 & 56126 & 331467 \\
\hline 398 & 1699 & 4718 & 39091 & 16681 & 164426 & 56601 & 208562 \\
\hline 434 & 3219 & 4737 & 149066 & 17222 & 1418027 & 58338 & 678737 \\
\hline 437 & 6278 & 4934 & 34755 & 17517 & 287966 & 60602 & 190297 \\
\hline 594 & 2771 & 5723 & 200658 & 17594 & 690795 & 60714 & 1250729 \\
\hline 619 & 2110 & 5934 & 321677 & 18289 & 76405 & 63441 & 4980197 \\
\hline 635 & 2394 & 6021 & 7622 & 19299 & 60602 & 66195 & 2110634 \\
\hline 874 & 1057 & 6038 & 398269 & 20229 & 56462 & 67005 & 17168654 \\
\hline 962 & 6289 & 6074 & 394065 & 20942 & 206829 & 72627 & 5585522 \\
\hline 981 & 9329 & 6278 & 117741 & 23478 & 1576637 & 73507 & 541322 \\
\hline 1209 & 3002 & 6786 & 58289 & 27018 & 259571 & 76405 & 319189 \\
\hline 1209 & 13205 & 6867 & 65246 & 27585 & 402194 & 78174 & 730835 \\
\hline 1251 & 3518 & 7371 & 8018 & 29379 & 421490 & & \\
\hline 1638 & 4619 & 7859 & 14934 & 32942 & 2080413 & & \\
\hline 2021 & 43897 & 8379 & 14258 & 37989 & 4701590 & & \\
\hline
\end{tabular}




\section{References}

[1] W. H. Mills, A system of quadratic diophantine equations, Pacific J. Math. 3 (1953), 217.

[2] S. P. Mohanty, A system of cubic diophantine equations, J. Number Theory 9 (1977), 153-159.

ROYAL INSTITUTE OF TECHNOLOGY

S-10044 STOCKHOLM, SWEDEN

Received on 24.12.1992

and in revised form on 31.3 .1993 